\title{
Eye position effects in macaque area V4
}

\author{
Frank Bremmer
}

Department of Zoology and Neurobiology, Ruhr-University Bochum, D-44780 Bochum, Germany

Received I4 December 1999; accepted II February 2000

\begin{abstract}
Acknowledgements: The author thanks Drs A. Thiele and P. Henning for helping to prepare the second monkey for the experiments, Dr C. Distler for her help with histology, and W. Zinke for data analysis. This work was supported by grants from the Deutsche Forschungsgemeinschaft (SFB 509/B7) and the Human Frontier Science Program (RG7I/96B).
\end{abstract}

Recent studies revealed an influence of eye position on neuronal discharges in many dorsal stream areas of the macaque visual cortical system. This eye position information is thought to serve an implicit non-retinocentric representation of visual spatial information. The question arises of whether the two visual cortical pathways encode information in a common coordinate system, i.e. whether eye position effects

Key words: Area V4; Coordinate transformation; Eye position; Monkey exist also in ventral stream areas. We recorded 112 neurons from area V4 of two awake monkeys. Of these, 55 (49\%) showed eye position effects. Like in dorsal stream areas, this modulatory influence was balanced out at the population level. Our data support the view of eye position effects as a general phenomenon in the macaque visual cortical system. NeuroReport I I:I277-I283 (C) 2000 Lippincott Williams \& Wilkins.

\section{INTRODUCTION}

Eye position effects are a common phenomenon in dorsal stream areas of the macaque visual cortical system [1-6]. Eye position effects are observed for stimulus driven activity, eye movement related activity (saccades and pursuit) as well as during pure fixation tasks, i.e. without further visual stimulation. Such a modulatory influence of the position of the eyes in the orbit usually does not affect the tuning properties of a cell. Instead, considering e.g. the preferred direction of visual stimulus motion, a change in eye position rather increases or decreases the cell's response for all stimulus directions. The percentage of cells influenced by eye position ranges from about $50 \%$ during pure fixation up to some $80 \%$ for visually induced or eye movement related activity. The eye position effect often is linear, i.e. neuronal discharge increases or decreases linearly with horizontal and/or vertical eye position $[7,8]$.

Interestingly, eye position effects are not only found in higher dorsal stream areas, but also at the very first stage of visual cortical processing $[9,10]$ as well as in premotor cortex [11,12] and the supplementary eye field [13]. As a general rule valid in all mentioned areas, eye position effects occur at the single cell level but are balanced out at the population level, i.e. the average discharge observed for a population of cells tested in a single paradigm is independent of eye position. This response feature of the ensemble of cells can be seen as prerequisite for the encoding of eye position at the population level [14]. The implicit encoding of eye position together with the information about the location of a stimulus on the retina could be used for the construction of a representation of visual spatial information in a non-retinocentric frame of reference. Such an implicit encoding originally had been sug- gested by theoretical studies on eye position effects [1517]. Two recent studies showed that the recorded activities from parietal and premotor areas are indeed suited to generate such an encoding of visual information in a coordinate system centered on a body part different from the eye $[8,18]$.

A transformation of visual signals into a non-retinocentric representation is thought to be essential for the sensorimotor system. As an example, reaching out for an object has to be performed in body rather than eye coordinates. However, particular properties of such an object, such as color and form, are encoded mainly in the ventral pathway of the macaque visual cortical system. The question thus arises of whether different properties of an object such as location with respect to the body and color are encoded in a common or in different frames of reference. To put it in other words: do such eye position effects exist only in the monkey's sensorimotor system, i.e. in dorsal stream areas, or are eye position effects a general phenomenon occurring in all cortical areas related to the processing of visual information? In order to answer this question we recorded neuronal activity in area V4 of the awake macaque monkey while animals fixated targets positioned at different locations on a screen. About half of the cells revealed an eye position effect during fixation in darkness. The characteristics of the modulatory influence of eye position were very similar to those already described for dorsal stream areas: neuronal discharge often varied linearly with respect to horizontal and/or vertical eye position, and it was at equilibrium for the population of cells. It thus seems as if the eye position effect is a common phenomenon throughout the monkey cortical system, probably subserving an implicit representation of 
spatial information in a non-retinocentric frame of reference.

\section{MATERIALS AND METHODS}

Experiments were performed in two male macaque monkeys (M. mulatta: $5.5 \mathrm{~kg}$ and $9.5 \mathrm{~kg}$, respectively). All animal care, housing, and surgical procedures were in accordance with national German and international published guidelines on the use of animals in research (European Communities Council Directive 86/609/ECC).

Animal preparation and recordings: Monkeys had been surgically prepared for recordings: under general anesthesia and sterile surgical conditions each animal had been implanted with a device for holding the head. A recording chamber for microelectrode penetrations through the intact dura was placed flat to the skull, a scleral search coil for monitoring eye position was implanted. Analgesics, antibiotics etc. were given postoperatively. Recording started 1 week after surgery. For cell recordings in the first monkey we used single electrodes (tungsten-in-glass electrodes, impedance $1-2 \mathrm{M} \Omega$ at $135 \mathrm{kHz}$ ) advanced by use of a hydraulic microdrive (Narishige) which was mounted on the recording chamber. For recordings in the second monkey we used a setup with multiple electrodes (Marburg Matrix, Eckhorn system, impedance 6-8 M $\Omega$ at $135 \mathrm{kHz}$ ). Histological analysis in both monkeys verified that recording sites had been located in area V4.

Behavioral paradigm and data analysis: During the experiments, animals were seated in a primate chair facing a translucent screen $48 \mathrm{~cm}$ in front of them and performing fixation tasks for liquid rewards. Rewards were given for keeping the eyes within an electronically defined window of $2 \times 2^{\circ}$ centered on the fixation target. The fixation target as well as the visual stimuli were back-projected onto the screen subtending the central $90 \times 90^{\circ}$ of the visual field.

In the first monkey we tested for an eye position effect during fixation in darkness. In these experiments, targets were back-projected in pseudo-randomized order at nine different locations on the screen. Locations were the center of the screen plus eight concentrically located points $15^{\circ}$ away from the center $\left(\mathrm{x}, \mathrm{y}=0^{\circ}, 0^{\circ} ; 0^{\circ}, \pm 15^{\circ} ; \pm 15^{\circ}, 0^{\circ} ; \pm 10.6^{\circ}\right.$, $\left.\pm 10.6^{\circ}\right)$. Presentation of fixation targets lasted for $1000 \mathrm{~ms}$ after the animal's eye position had been located continuously within an electronically defined window for at least $300 \mathrm{~ms}$. Mean neuronal activity was computed for each fixation location during this $1000 \mathrm{~ms}$ epoch. In the second monkey, we tested for an eye position effect during fixation in darkness at one of five target locations $\left(\mathrm{x}, \mathrm{y}=0^{\circ}, 0^{\circ} ; \pm 10^{\circ}, \pm 10^{\circ}\right)$. Differences in activity were tested for statistical significance with a distribution-free ANOVA. Two-dimensional linear regression analysis was applied to quantify the eye position effect. For validating the planar model as fit to the observed data, an F-test was employed.

Some cells were also tested for an effect of eye position on stimulus driven activity. In the first monkey, we used a technique described in detail in [5]. Briefly, while the monkey fixated the target an optimized stimulus (oriented light bar) was flashed across the RF. During fixation, the monitored eye signal was superimposed onto the signal driving the stimulus to guarantee stimulation of the same part of the retina each time (retinal clamp). In the second monkey we mapped the location of the visual RF using a technique slightly modifed from that described in [19]. In this paradigm, the central $60 \times 60^{\circ}$ of the visual field were subdivided into a virtual grid of 36 (six horizontal $\times$ six vertical) possible stimulus locations $\left(10^{\circ}\right.$ square subregions). During a single fixation we presented six brief, nonoverlapping visual stimuli (200 ms each) separated by nonstimulus periods of $200 \mathrm{~ms}$. Stimuli were stationary $10^{\circ}$ square patches covering a single grid subregion. Thus six trials each were necessary to map a receptive field completely. Usually 6-10 replications of such a complete RF map were performed during a single experiment. RF maps were constructed off-line by counting the total number of spikes evoked by stimulating a given grid subregion using a shifted temporal window adjusted to the cell's response latency. Spike count was averaged over a number of replications and converted into mean firing rates. The resulting $6 \times 6$ raw data matrix was subsequently converted into a $21 \times 21$ matrix by linear interpolation of three new points between each original data point and plotted as a pseudo-color map.

Many neurons in area V4 are wavelength selective [20]. In order to optimize the visual stimulus we mapped a cell's visual receptive field in a first step during central fixation with four different isoluminant stimuli $\left(1 \mathrm{~cd} / \mathrm{m}^{2}\right)$ : three narrow-band colored and one white. Visual stimuli were generated by a Silicon Graphics Workstation (Indigo2, High Impact) and presented by a video projector (Electrohome 4300). The narrow-band colored lights were generated by the use of different spectral filters. Spectral bandwidth and wavelength peak of the different colored lights were measured with a spectrophotometer: (i) red: $570-660 \mathrm{~nm}$ with a peak at $600 \mathrm{~nm}$, (ii) green: $515-555 \mathrm{~nm}$ with a peak at $530 \mathrm{~nm}$, (iii) blue: $390-490 \mathrm{~nm}$, with a peak at $430 \mathrm{~nm}$. From these four stimuli we selected the one eliciting the strongest neuronal response. This optimized visual stimulus was used in a second step to test for an eye position effect on visual driven activity. To this end, we mapped RFs for five different fixation locations $\left(x, y=0^{\circ}, 0^{\circ}\right.$; $\pm 10^{\circ}, \pm 10^{\circ}$. Fixation locations varied in pseudo-randomized order from trial to trial. Analysis concerning a possible eye position effect was based on the number of spikes in the post-stimulus window for all grid locations evoking discharges $>50 \%$ of the maximum firing rate.

\section{RESULTS}

The eye position effect during fixation in darkness: single cell level: A total of 112 neurons from two monkeys was recorded during fixation in darkness: 73 neurons from the right hemisphere of the first monkey and 39 neurons from the right hemisphere of the second monkey. Activity of about half the cells $(55 / 112=49 \%: 36 / 73=49 \%$ in the first monkey, $19 / 39=48 \%$ in the second monkey) was influenced by the different positions of the eyes in the orbit during fixation in darkness. An example of such an eye position effect on a cell's discharge is shown in Fig. 1. The histogram in the left panel (Fig. 1a) shows mean ( \pm s.d.) discharge values for the different fixation locations. For this individual cell, discharges were strongest for fixations right and downward (ANOVA: $p<0.001$ ). Neuronal activity decreased for fixations up and leftward. In order to 
Single cell level

(a)

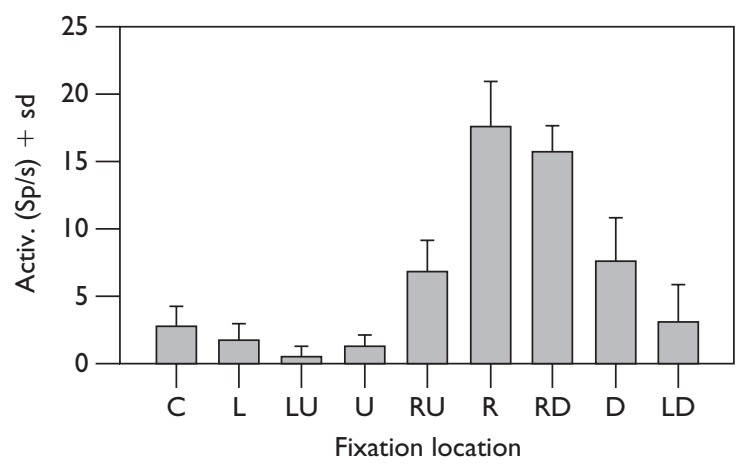

unit frol4

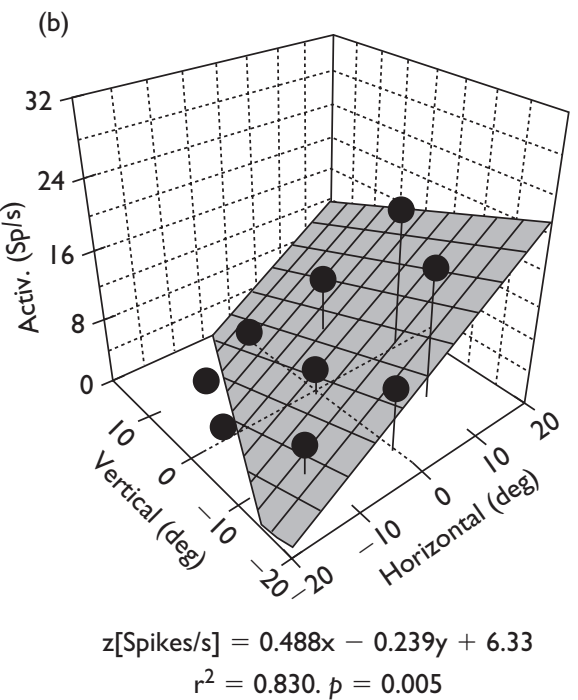

Fig. I. Eye position effect during fixation in darkness: single cell level. (a) Mean activity values ( \pm s.d.) observed at nine different locations (C, center; LU, left up; U, up; RU, right up; RD, right down; D, down; LD, left down; $L$, left). For this neuron, discharge rates were strongest for fixation locations mostly to the right (ANOVA $p<0.00 \mathrm{I}$ ). (b) The shaded plane represents the two-dimensional linear regression to the mean discharge values $\left(r^{2}=0.830, p=0.005\right)$. The $x-y$ plane in this plot represents the central $40 \times 40^{\circ}$ of the tangent screen. The base point of each drop line depicts the fixation location on the screen and the height of each line depicts the mean activity value during fixation at this location.

quantify this modulatory influence we employed twodimensional linear regression analysis. The result of this computation is shown in the panel on the right (Fig. 1b). The $x-y$-base of this cube represents the screen in front of the monkey. The needle-like drop lines show essentially the same data as in Fig. 1a, now in a 3-D view. The base point of each drop line indicates the fixation location on the screen. The shaded plane depicts the regression plane approximated to the mean discharge values, with regression parameters given below the diagram. For this cell the linear model proved to be statistically significant (multiple linear regression, $p=0.005)$.

The eye position effect for visual driven activity: A few cells $\left(n=12: \mathrm{n}_{1}=4\right.$ cells from the first monkey and $\mathrm{n}_{2}=8$ cells from the second monkey) revealing an eye position effect during fixation in darkness were also tested with visual stimuli. All of these cells also revealed an eye position effect on their visual driven activity. An example is shown in Fig. 2. In a first step this neuron was tested for its spectral sensitivity with isoluminant stimuli of different wavelengths. During central fixation, the receptive field was mapped with a procedure explained in detail in the Materials and Methods. This neuron revealed a clear spectral preference for a green visual stimulus (Fig. 2a). This stimulus was used to map the receptive field while the monkey fixated at five different locations (Fig. 2b). As can be seen, the receptive field moved with the fixation location, i.e. this neuron, as all others tested, had an eye centered visual receptive field. However, the maximum response strength varied with eye position: strongest discharge was obtained for fixation locations above the horizontal meridian (ANOVA: $p<0.0001$ ).
The eye position effect during fixation in darkness: population level: A regression plane was approximated to the discharge of each individual neuron with an eye position effect. For $44 \%$ of these neurons, this fit was significant at $p<0.05$. For another $17 \%$ of the neurons, the approximation was nearly significant at $p<0.1$. The eye position effect, which could be observed at the single cell level, was at equilibrium at the population level. Figure $3 a, b$ shows the values for the first animal: cells were tested at nine different fixation locations. The histogram (Fig. 3a) shows the mean ( \pm s.d.) discharge rate of those cells whose discharge was significantly influenced by eye position $(n=36)$. Average discharge values of the population of neurons for the different fixation locations were not significantly different (ANOVA: $\mathrm{H}=0.708, \mathrm{DF}=8, p>0.999$ ). An average response plane was approximated to the ensemble response. As shown in Fig. 3b, this response plane was essentially flat, indicating again the unbiased discharge characteristic at the population level. The same result was obtained from the population of neurons from the second animal (Fig. 3c,d; ANOVA: $\mathrm{H}=0.578, \mathrm{DF}=4$, $p>0.95)$.

\section{DISCUSSION}

Eye position effects in ventral stream areas: Recently, eye position effects have been described for the earliest stage of cortical processing of visual information, i.e. area V1 $[9,10]$. Different substructures within area V1 feed into different parts of the visual cortical system, i.e. cells from blob and interblob regions project into the ventral stream whereas cells outside these blobs project into the dorsal stream. Although extremely unlikely, it could had been the case that V1 neurons revealing an eye position effect were 
(a)

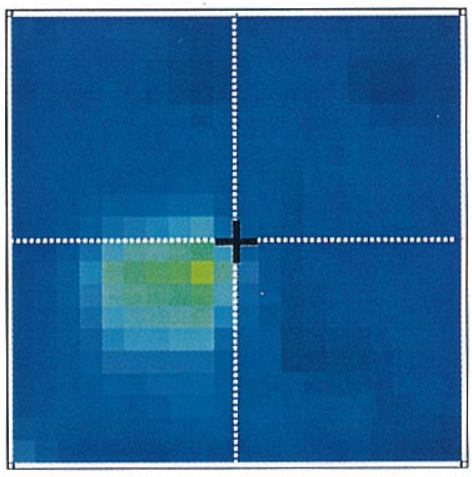

RED

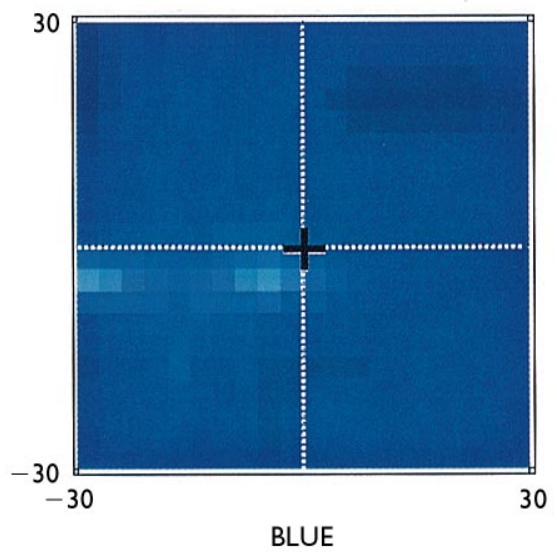

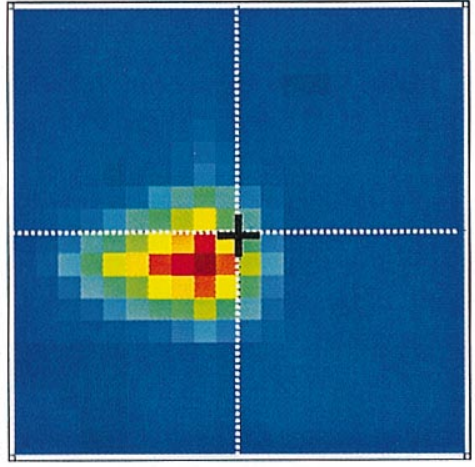

GREEN
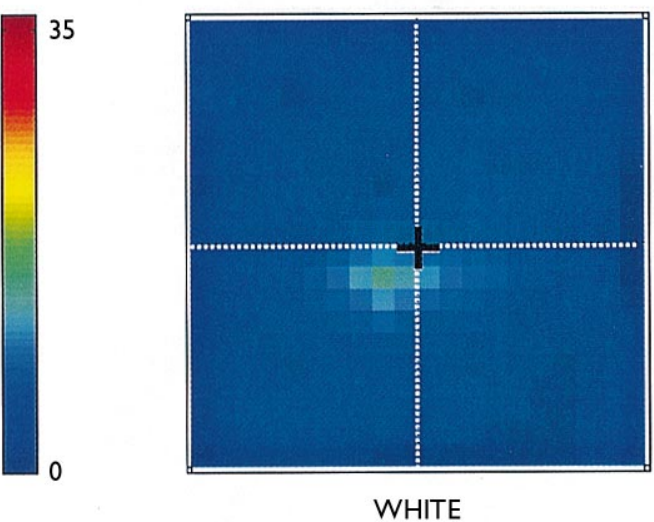

Fig. 2. (a) Receptive field mapping. During central fixation this neuron's receptive field was mapped with four isoluminant stimuli (see Materials and Methods for details). Each panel indicates the receptive field map $\left( \pm 30^{\circ}\right.$ in the horizontal and vertical domain) obtained by presentation of the respective colored light stimulus. The white dotted lines within each panel indicate the horizontal and vertical meridian on the projection screen. The black cross indicates the fixation location. Neuronal activity is color coded, with red corresponding to high and blue corresponding to low discharge rates. The color code scales to the maximum discharge across all four RF maps. This neuron clearly preferred the green stimulus. (b) RF mapping for five different fixation locations. Using the optimized green visual stimulus, the neuron's receptive field was mapped while the monkey fixated at five different locations. Dotted lines, black cross and color coding as in (a). Each panel represents the RF map obtained at a specific location: e.g. the upper left panel shows the RF map for upper left fixation, etc. Neuronal activity was profoundly influenced by eye position: strongest discharges were observed for fixation locations above the horizontal meridian (ANOVA: $p<0.001$ ).

exclusively located outside the blob regions and therefore would have been classified as dorsal stream neurons. In our present study we therefore recorded neurons from area $\mathrm{V} 4$, i.e. the major gateway of the ventral stream of the visual cortical system. As in many dorsal stream areas, about half of the cells in area V4 revealed an influence of eye position on their discharge during fixation in darkness. Eye position effects were balanced out for the population of cells. In addition, all the cells with an eye position effect in darkness revealed also such an effect on their stimulus driven activity. The functional properties of eye position effects in area V4 are therefore very similar to those found in dorsal stream areas.

Eye position effects in dorsal stream areas: The dorsal stream of the macaque visual cortical system is thought to deal especially with the processing of spatial information. In humans as well as non-human primates, lesions of this part of the brain (posterior parietal cortex: PPC) can lead to a severe impairment in visual spatial perception within the visual hemifield contralateral to the lesion site, better known as hemineglect [21-23]. This neglect usually occurs with respect to the head or the body rather than the eye, i.e. visual deficits are organized not with respect to the receptor epithelium (the retina) but rather in a higher order coordinate system. These and other experimental data led to the hypothesis that one of the functions of the PPC might be the transformation of visual signals into a nonretinocentric (e.g. head-centered) frame of reference. In a pioneering study, Andersen and Mountcastle showed that the activity of a substantial portion of neurons within monkey PPC was modulated by gaze direction [24]. It was argued that such eye position information might be used to generate a head-centered encoding of visual spatial information [15]. In the following years, eye position effects were shown to exist in almost all areas of the dorsal stream 
(b)
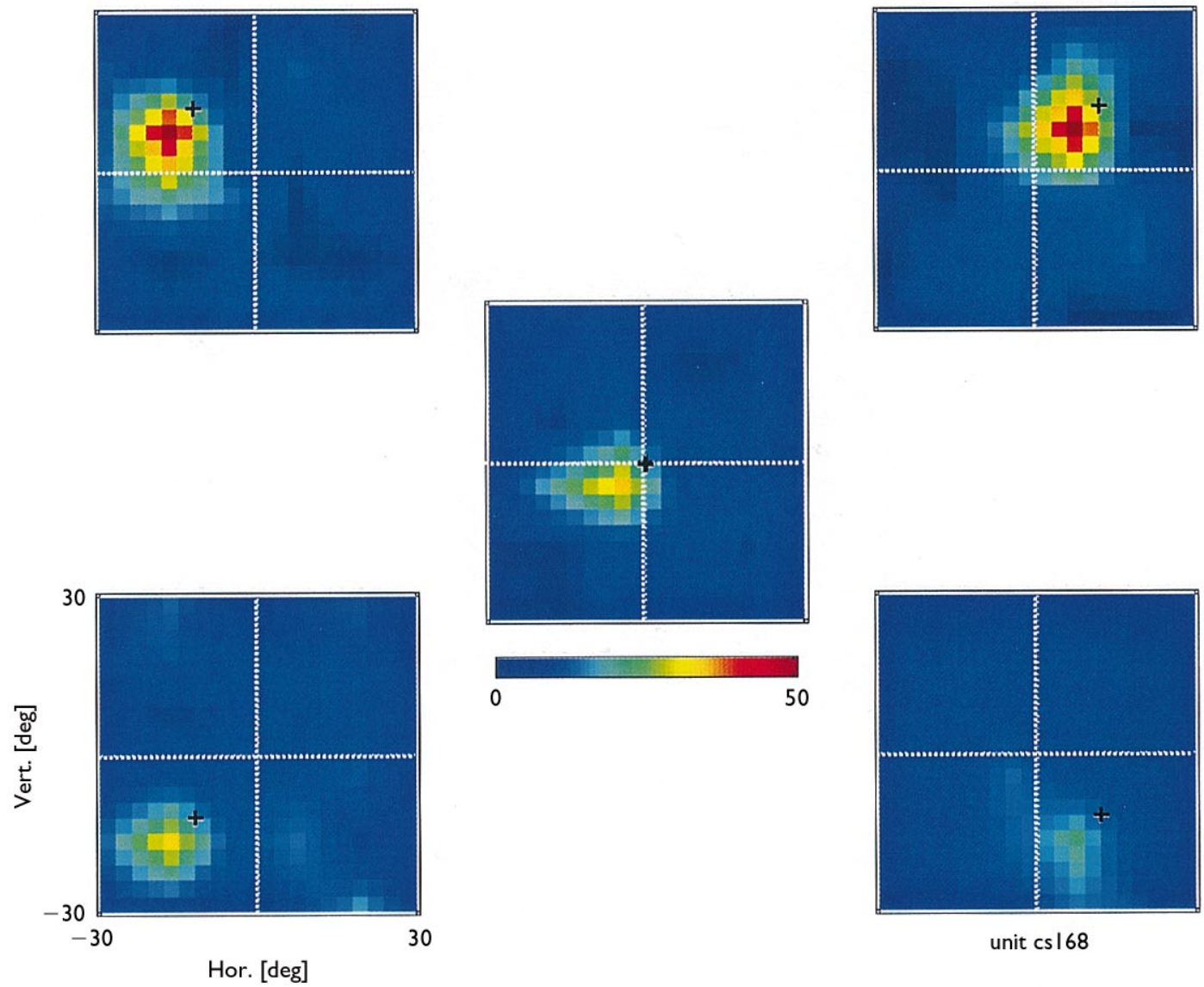

unit cs 168

Fig. 2. (Continued).

of the macaque visual cortical system [2-7]. Finally, eye position effects were also found in premotor structures $[12,13,25]$. All these findings were taken as evidence for the hypothesis of a coordinate transformation of visual signals in a non-retinocentric frame of reference within the cortical sensorimotor pathway.

Eye position effects as general phenomenon in the monkey visual cortical system: Our data argue against such a uniqueness of eye position effects in dorsal stream areas. Rather, they provide evidence for our hypothesis that the modulatory influence of eye position on neuronal discharges is a common phenomenon throughout the monkey cortical system. Two recent studies could show that the distribution of eye position effects found in dorsal stream areas is suited to generate the hypothesized non-retinocentric, possibly craniocentric encoding of visual spatial information $[8,18]$. Such encoding would be implicit, i.e. a population of cells would be necessary to accomplish this computational step. Our data clearly indicate that such an implicit head-centered encoding would also be possible in area V4. This would mean that visual information concerning object properties such as (i) color (ventral stream) and (ii) location (dorsal stream) could be encoded simultaneously in the same spatial frame of reference across different cortical areas. It therefore seems as if the cortex uses a general purpose, i.e. implicit representation of spatial information in a head-centered frame of reference across areas and different visual cortical streams. In parallel, a special purpose, i.e. explicit head-centered encoding at the single cell level like e.g. in area VIP [19] is present only in areas which participate specifically in the control and sensory guidance of body parts other than the eyes.

\section{CONCLUSION}

The modulatory influence of the position of the eyes in the orbit on neuronal discharges is not restricted to areas of the dorsal stream of the macaque visual cortical system. Our data clearly show that an eye position effect can also be found in ventral stream area V4. In addition, previous studies also showed an eye position effect in striate cortex. Finally, even discharges of neurons in the supplementary eye field and the premotor cortex are influenced by eye position. We thus consider the modulatory influence of eye position on neuronal discharges to be a common phenomenon throughout the monkey cortical system. Discharge properties are suited for an implicit representation of spatial information in a non-retinocentric frame of reference. The generality of the eye position effect could allow for a consistent visual spatial encoding across different ventral and dorsal stream areas. 
Population level

(a)

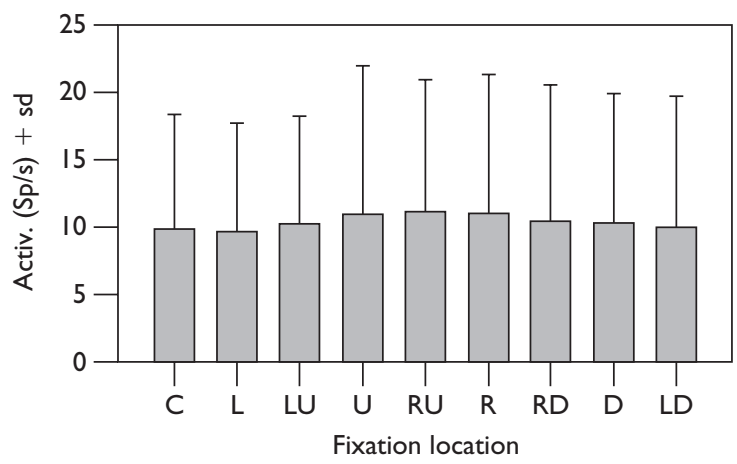

(c)

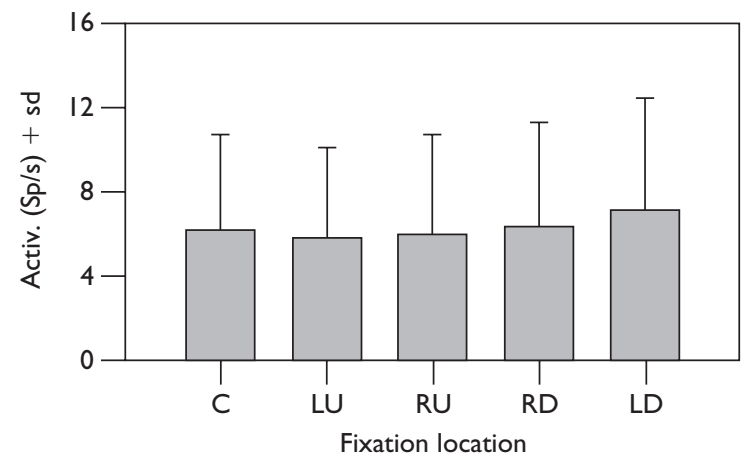

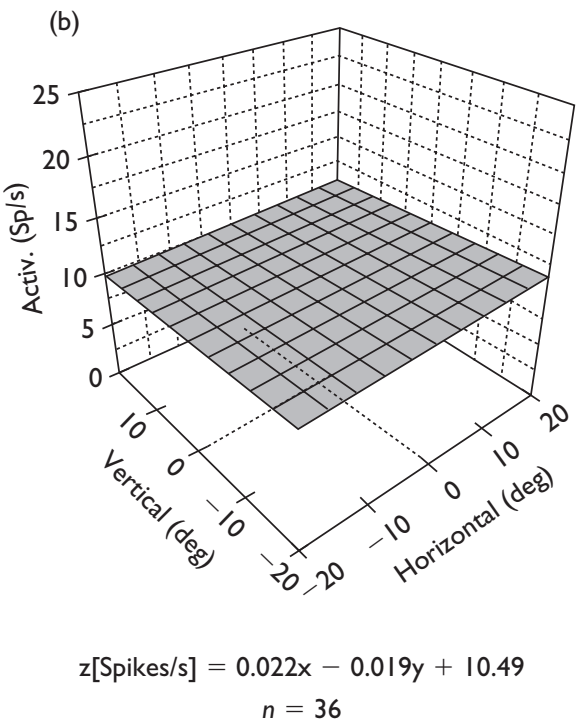

(d)

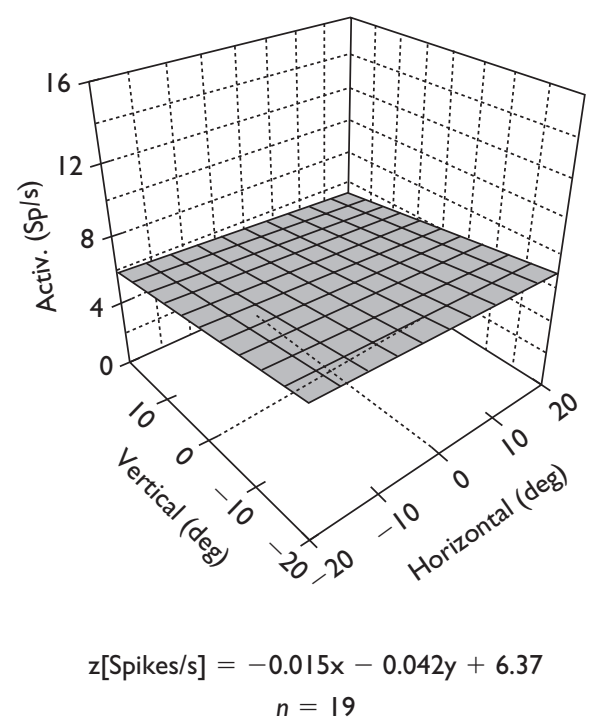

Fig. 3. Eye position effect during fixation in darkness: population level. (a,c) Mean activity ( \pm s.d.) for nine (a) and five (c) differnet eye positions, respectively (abbreviations as in Fig. I). The discharge values for the different eye positions were not significantly different (ANOVA: $p<0.9$ in both cases), indicating that the modulatory effect of eye position is balanced out at the population level. (b,d) The mean population response planes were obtained by averaging the cell reponses of the eye position affected neurons and approximating the regression plane to these discharge values. The resulting discharge planes proved to be flat.

\section{REFERENCES}

1. Andersen RA, Essick GK and Siegel RM. Science 230, 456-458 (1985).

2. Galletti C and Battaglini PP. J Neurosci 9, 1112-1125 (1989).

3. Galletti C, Battaglini PP and Fattori P. Eur J Neurosci 7, 2486-2501 (1995)

4. Bremmer F, Distler C and Hoffmann KP. J Neurophysiol 77, 962-977 (1997).

5. Bremmer F, Ilg UJ, Thiele A et al. J Neurophysiol 77, 944-961 (1997).

6. Bremmer F, Graf W, Ben Hamed S and Duhamel JR. Neuroreport 10 873-878 (1999).

7. Andersen RA, Bracewell RM, Barash S et al. J Neurosci 10, 1176-1196 (1990).

8. Bremmer F, Pouget A and Hoffmann KP. Eur J Neurosci 10, 153-160
(1998).

9. Guo K and Li CY. Neuroreport 8, 1405-1409 (1997).

10. Trotter Y and Celebrini S. Nature 398, 239-242 (1999).

11. Boussaoud D. J Neurophysiol 73, 886-890 (1995).

12. Boussaoud D, Jouffrais C and Bremmer F. J Neurophysiol 80, 1132-1150 (1998).

13. Schlag J, Schlag-Rey M and Pigarev I. Exp Brain Res 90, 302-306 (1992).

14. Sanger TD. Neurol Comp 6, 29-37 (1994).

15. Zipser D and Andersen RA. Nature 331, 679-684 (1988).

16. Mazzoni P, Andersen RA and Jordan MI. Proc Natl Acad Sci USA 88, 4433-4437 (1991).

17. Pouget A, Fisher SA and Sejnowski TJ. J Cogn Neurosci 5, 150-161 (1993).

18. Boussaoud D and Bremmer F. Exp Brain Res 128, 170-180 (1999). 
19. Duhamel JR, Bremmer F, Ben Hamed S and Graf W. Nature 389, 845848 (1997).

20. Schein SJ and Desimone R. J Neurosci 10, 3369-3389 (1990).

21. Andersen RA and Gnadt JW. Rev Oculomotor Res 3, 315-335 (1989).
22. Driver J and Mattingley J Nature Neurosci 1, 17-22 (1998)

23. Mesulam MM. Phil Trans R Soc Lond B, Biol Sci 354, 1325-1346 (1999).

24. Andersen RA and Mountcastle VB. J Neurosci 3, 532-548 (1983).

25. Boussaoud D, Barth TM and Wise SP. Exp Brain Res 93, 423-434 (1993). 